\title{
Constrained and unconstrained rearrangement minimization problems related to the p-Laplace operator
}

\author{
Behrouz Emamizadeh \\ Faculty of Science and Engineering \\ The University of Nottingham Ningbo China \\ 199 Taikang East Road, Ningbo, Zhejiang China 315100 \\ E-mail: Behrouz.Emamizadeh@nottingham.edu.cn \\ Telephone number: 0086-512-88161368 \\ Fax number: 0086-512-81880442 \\ Yichen Liu \\ Department of Mathematical Sciences \\ Xi' an Jiaotong-Liverpool University \\ Suzhou Industrial Park, Suzhou, Jiangsu China 215123 \\ E-mail: Yichen.Liu@liverpool.ac.uk
}

\begin{abstract}
In this paper we consider an unconstrained and a constrained minimization problem related to the boundary value problem

$$
-\Delta_{p} u=f \quad \text { in } D, \quad u=0 \quad \text { on } \partial D .
$$

In the unconstrained problem we minimize an energy functional relative to a rearrangement class, and prove existence of a unique solution. We also consider the case when $D$ is a planar disk and show that the minimizer is radial and increasing. In the constrained problem we minimize the energy functional relative to the intersection of a rearrangement class with an affine subspace of codimension one in an appropriate function space. We briefly discuss our motivation for studying the constrained minimization problem.
\end{abstract}

Key Words: Minimization, Rearrangement theory, Existence, Uniqueness, Radial solutions, subdifferentials

Mathematics Subject Classification: 35J20, 35J25, 65K10 


\section{Introduction}

Consider the boundary value problem

$$
\begin{cases}-\Delta u+h(x) u=f(x) & \text { in } D \\ u=0 & \text { on } \partial D\end{cases}
$$

where $D$ is a smooth ( $C^{2}$ is enough) bounded domain in $\mathbb{R}^{2}$. The functions $h(x)$ and $f(x)$ are non-negative and bounded. Physically, (1.1) models an elastic membrane which is fixed around the boundary, subject to a vertical force $f(x)$. The function $h(x)$ represents the density of the membrane, and $u$ the displacement from the rest position. In case the membrane is isotropic, i.e. it is made of a single material, $h=0$, hence (1.1) reduces to the classical Poisson's problem:

$$
\begin{cases}-\Delta u=f(x) & \text { in } D \\ u=0 & \text { on } \partial D .\end{cases}
$$

The energy functional associated with (1.2) is defined by

$$
\Phi(f)=\int_{D} f u_{f} d x
$$

where $u_{f} \in H_{0}^{1}(D)$ is the unique positive solution of (1.2). Two interesting optimization problems related to $\Phi$ are as follows:

$$
\sup _{f \in \mathcal{R}} \Phi(f) \quad \text { and } \quad \inf _{f \in \mathcal{R}} \Phi(f)
$$

where $\mathcal{R}$ denotes a rearrangement class generated by a known function, see section 2 for precise definitions. Both of these problems have been extensively investigated by G. R. Burton in [1], [2] and [3]. In recent years a number of mathematicians have attempted to apply the tools introduced by Burton to various optimization problems similar to the ones mentioned above. The present paper is a work in this direction.

Let us now proceed to describe precisely the problems that will be discussed here. First, we consider the following boundary value problem:

$$
\begin{cases}-\Delta_{p} u=f & \text { in } D \\ u=0 & \text { on } \partial D\end{cases}
$$

where $\Delta_{p}$ is the classical $p$-Laplace operator, i.e. $\Delta_{p} u=\nabla \cdot\left(|\nabla u|^{p-2} \nabla u\right)$, with $p>1$. Next, denoting the unique solution of (1.4) by $u_{f} \in W_{0}^{1, p}(D)$, and recalling that $u_{f}$ is the unique minimizer of the functional

$$
F(u)=\frac{1}{p} \int_{D}|\nabla u|^{p} d x-\int_{D} f u d x
$$


relative to $u \in W_{0}^{1, p}(D)$, we define the $p$-energy functional associated to (1.4), as follows:

$$
\Phi_{p}(f)=\int_{D} f u_{f} d x
$$

We are interested in the following optimization problems:

$$
\inf _{f \in \mathcal{R}} \Phi_{p}(f)
$$

and

$$
\inf _{f \in \mathcal{R} \cap \Lambda} \Phi_{p}(f),
$$

where $\mathcal{R}$ denotes a class of rearrangements generated by a known function, and $\Lambda$ an affine subspace of codimension one in an appropriate function space.

Let us describe the physical interpretation of (1.6) which is most realistic when $p=2$. The goal is to identify a force function selected from $\mathcal{R}$, in such a way that the total displacement of the membrane is as small as possible. A similar problem has been considered in [10]. In that paper the authors considered an elastic membrane made out of two materials with prescribed quantities, subject to a fixed vertical force. They proved the existence of the best possible design so that the corresponding total displacement is minimal. The analysis conducted in [10] was based on tangent cones, but in the present work we follow the approach of [14], [1], [2] and [3].

The physical relevance of (1.7) can be described similarly to the unconstrained problem. In this case, we are interested in minimizing the total displacement of the membrane under the constraint that the vertical force is admissible provided it is applied to a location intersecting a prescribed set.

Problem (1.6) has been considered in [14], under very restrictive conditions on the generator of the rearrangement class. More precisely, the author imposed the generator to be strictly positive and bounded. In this paper, we remove both of these conditions. In addition, we address the case where $D$ is a ball, an interesting situation that is neglected in [14]. In [7], the authors discussed the maximization version of (1.6); that is,

$$
\sup _{f \in \mathcal{R}} \Phi_{p}(f) .
$$

Motivated by [7], the paper [8] mainly discusses a maximization problem related to the following boundary value problem:

$$
\begin{cases}-\Delta_{p} u+|u|^{p-2} u=0 & \text { in } D \\ |\nabla u|^{p-2} \frac{\partial u}{\partial \nu}=f(x) & \text { on } \partial D\end{cases}
$$

where $f \in \mathcal{R}$, and $\frac{\partial}{\partial \nu}$ denotes the outward normal derivative to the boundary. More precisely, the authors investigate the following maximization problem:

$$
\sup _{f \in \mathcal{R}} \mathcal{I}(f)
$$


where

$$
\mathcal{I}(f)=\int_{\partial D} f u_{f} d \mathcal{H}^{n-1}
$$

Here $u_{f} \in W^{1, p}(D)$ denotes the unique solution of (1.8), and $d \mathcal{H}^{n-1}$ stands for the $(n-1)$ dimensional Hausdorff measure on $\partial D$.

We also mention that the problem considered in [10] can also be treated using the techniques implemented in the present paper. Indeed, the analysis employed in [10] is based on the notion of tangent cones, a tool which would not be applicable in a wide range of applications. In particular, if one intends to construct a membrane, as described in [10], out of three or more materials, then the method of tangent cones will definitely fail, but the method of rearrangements can successfully be applied.

Problem (1.7), to the best of our knowledge is new. We hope it will serve as a motivation for further research. Henceforth, we refer to (1.6) as the unconstrained problem, and (1.7) as the constrained problem.

The rest of the paper is organized as follows. In section 2, we review some materials from the rearrangement theory, and recall some results from functional analysis. Section 3, is devoted to the unconstrained problem (1.6). In that section we also consider the case of $D$ being a planar disk, and prove that the minimizer is radial and increasing. In section 4 , we consider the constrained problem (1.7), and show there exists a unique solution. Because of the presence of the constraint in this problem, the expectation of having a radially increasing minimizer, in case the domain is a disk, is no longer guaranteed.

\section{Preliminaries}

This section gathers the background for the sections to follow. We begin by reviewing the relevant parts of the rearrangement theory attributed to G. R. Burton. The appropriate references for this section are [1], [2] and [12]. We stress that the materials to follow are specialized to suit the purpose of the present paper, hence they may not appeal in the most generality.

Definition 2.1. Let $X$ and $X^{\prime}$ be two measurable subsets of $\mathbb{R}^{N}$ and $\mathbb{R}^{M}$, respectively. Suppose $\mu_{N}(X)=\mu_{M}\left(X^{\prime}\right)<\infty$, where $\mu_{N}$ and $\mu_{M}$ denote the Lebesgue measures in $\mathbb{R}^{N}$ and $\mathbb{R}^{M}$, respectively. Suppose $f: X \rightarrow[0, \infty)$ and $g: X^{\prime} \rightarrow[0, \infty)$ are measurable functions. We say $f$ and $g$ are rearrangements of each other if:

$$
\lambda_{f, \mu_{N}}(\alpha) \equiv \mu_{N}(\{x \in X: f(x) \geq \alpha\})=\mu_{M}\left(\left\{x \in X^{\prime}: g(x) \geq \alpha\right\}\right) \equiv \lambda_{g, \mu_{M}}(\alpha), \quad \forall \alpha \geq 0 .
$$

Definition 2.2. Let $f$ be a function as in Definition 2.1. The function $f^{\Delta}:\left(0, \mu_{N}(X)\right) \rightarrow \mathbb{R}$ defined by

$$
f^{\Delta}(s)=\max \left\{\alpha: \lambda_{f, \mu_{N}}(\alpha) \geq s\right\}
$$

is called the decreasing rearrangement of $f$. Also, the function $f_{\Delta}(s) \equiv f^{\Delta}\left(\mu_{N}(X)-s\right)$ is called the increasing rearrangement of $f$. 
The following remark is useful.

Remark 2.1 It is well known that when $f$ is continuous and its graph has no significant flat zones in the sense that

$$
\mu_{N}(\{x \in X: f(x)=c\})=0, \quad \forall c \in \mathbb{R}^{+},
$$

then $f^{\Delta}$ and $f_{\Delta}$ will be both continuous, moreover, $f^{\Delta}$ will be strictly decreasing, and $f_{\Delta}$ will be strictly increasing.

Definition 2.3. Let $f$ be as in Definition 2.1. The set $\mathcal{R}(f)$, called the rearrangement class generated by $f$, is defined as follows:

$$
\mathcal{R}(f)=\{g: X \rightarrow[0, \infty): g \text { and } f \text { are rearrangements of each other }\} .
$$

One of the cornerstones in Burton's rearrangement theory is the following result.

Lemma 2.1. Let $p>1$, and $p^{\prime}$ be the conjugate exponent of $p$, i.e. $1 / p+1 / p^{\prime}=1$. Suppose $f \in L^{p^{\prime}}(X)$, and $\mathcal{R} \equiv \mathcal{R}(f)$ is the rearrangement class generated by $f$. Then

(i) $\mathcal{R} \subseteq L^{p^{\prime}}(X)$, and $\|f\|_{p^{\prime}}=\|g\|_{p^{\prime}}$, for every $g \in \mathcal{R}$. Here $\|\cdot\|_{p^{\prime}}$ denotes the usual $L^{p^{\prime}}$-norm.

(ii) $\overline{\mathcal{R}}$, the weak closure of $\mathcal{R}$ in $L^{p^{\prime}}(X)$, is convex and weakly compact in $L^{p^{\prime}}(X)$.

(iii) For $\mathcal{A}$ an affine subspace of finite codimension in $L^{p^{\prime}}(X)$, ext $(\overline{\mathcal{R}} \cap \mathcal{A})$, the set of extreme points of $\overline{\mathcal{R}} \cap \mathcal{A}$, is equal to $\mathcal{R} \cap \mathcal{A}$.

(iv) Let $\mathcal{A}$ be as in (iii). Then $\overline{\mathcal{R}} \cap \mathcal{A}=\overline{c o}(\mathcal{R} \cap \mathcal{A})$, the closed convex hull of $\mathcal{R} \cap \mathcal{A}$.

Proof. For (i) and (ii), see [1] and [2]. For (iii) and (iv), see [4].

Lemma 2.2. Let $f: X \rightarrow(0, \infty)$ and $g: X \rightarrow(0, \infty)$ be measurable functions. Suppose the graph of $g$ has no significant flat zones. Then there is a decreasing function $\phi$ such that $\phi \circ g$ and $f$ are rearrangements of each other. In particular,

$$
\phi(s)=f_{\Delta} \circ \lambda_{g, \mu_{N}}(s) .
$$

Proof. The proof is similar to Lemma 2.9 in [2]. Recalling Remark 2.1, we infer $g^{\Delta}$ is strictly decreasing, hence it has a left inverse which coincides with $\lambda_{g, \mu_{N}}$. We set $\phi(s)=f_{\Delta} \circ \lambda_{g, \mu_{N}}(s)$. To see that $\phi \circ g$ and $f$ are rearrangements of each other we first observe that $\phi \circ g$ and $\phi \circ g^{\Delta}$ are rearrangements of each other. This, in turn, implies $(\phi \circ g)_{\Delta}=\left(\phi \circ g^{\Delta}\right)_{\Delta}$. However, $\left(\phi \circ g^{\Delta}\right)_{\Delta}=\phi \circ g^{\Delta}$, hence $(\phi \circ g)_{\Delta}=\phi \circ g^{\Delta}=f_{\Delta} \circ \lambda_{g, \mu_{N}} \circ g^{\Delta}=f_{\Delta}$. Therefore, $\phi \circ g$ and $f$ are rearrangements of each other, as desired.

Lemma 2.3. Let $f \in L^{p^{\prime}}(X)$ and $g \in L^{p}(X)$ be non-negative functions, where $1 / p+1 / p^{\prime}=1$. Let $\mathcal{R}$ be the rearrangement class generated by $f$. Suppose there is a decreasing function $\phi$ such that $\phi \circ g \in \mathcal{R}$. Then $\phi \circ g$ is the unique minimizer of the linear functional

$$
L(h)=\int_{X} h g d \mu_{N},
$$

relative to $h \in \overline{\mathcal{R}}$. 
Proof. The proof is a minor variant of the proof of Lemma 2.4 in [3].

Lemma 2.4. Let $f \in L^{p^{\prime}}(X)$ and $\mathcal{R}$ be the rearrangement class generated by $f$. Let $\overline{\mathcal{R}}$ be the weak closure of $\mathcal{R}$ in $L^{p^{\prime}}(X)$. Then

$$
\overline{\mathcal{R}}=\left\{g \in L^{1}(X): \int_{X} g d \mu_{N}=\int_{X} f d \mu_{N} \quad \text { and } \int_{0}^{s} g^{\Delta} d t \leq \int_{0}^{s} f^{\Delta} d t, \forall s \in\left(0, \mu_{N}(X)\right)\right\} \text {. }
$$

Proof. See Lemma 2.2 in [3], or [17].

Corollary 2.5. Suppose the hypotheses of Lemma 2.4 hold. Let $h \in \overline{\mathcal{R}}$, and $\mathcal{R}(h)$ denote the rearrangement class generated by $h$. Then, $\mathcal{R}(h)$ is contained in $\overline{\mathcal{R}}$.

Proof. The proof follows immediately from Lemma 2.4 .

We will also need two results from functional analysis.

Lemma 2.6. Suppose $p>1$ and $p^{\prime}$ is the conjugate exponent of $p$. Suppose $f_{n} \rightarrow f$ in $L^{p^{\prime}}(X)$, and $g_{n} \rightarrow g$ in $L^{p}(X)$. Then $\int_{X} f_{n} g_{n} d \mu_{N} \rightarrow \int_{X} f g d \mu_{N}$.

Proof. The proof is straightforward, hence omitted.

Lemma 2.7. Let $\mathcal{C}$ be a convex set in a real vector space $Y$. Let $l_{1}$ and $l_{2}$ be linear functionals on $Y$, and $I$ be a real number for which there exist $y_{1}$ and $y_{2}$ in $\mathcal{C}$ such that $l_{1}\left(y_{1}\right)<I<l_{1}\left(y_{2}\right)$. Moreover, suppose there exists $y_{0} \in Y$ such that $l_{2}(y) \geq l_{2}\left(y_{0}\right)$, for all $y \in \mathcal{C}$ satisfying $l_{1}(y)=I$. Then there is a real number $\gamma$ such that $y_{0}$ minimizes $l_{2}+\gamma l_{1}$, relative to $\mathcal{C}$.

Proof. The proof is a minor variant of the proof of Lemma 2.13 in [2].

We end this section by recalling the notion of subdifferentiability of convex functionals, see for example [5].

Definition 2.4. For $r>1$, let $\Psi: L^{r}(X) \rightarrow \overline{\mathbb{R}}$ be a convex functional. We assume $\Psi$ is proper, i.e. $\Psi\left(u_{0}\right)<+\infty$, for some $u_{0} \in L^{r}(X)$ and nowhere takes the value $-\infty$. For $u \in L^{r}(X)$, the subdifferential of $\Psi$ at $u$ is denoted $\partial \Psi(u)$, and defined as follows:

$$
\partial \Psi(u)=\left\{w \in L^{r^{\prime}}(X): \Psi(v) \geq \Psi(u)+\int_{X}(v-u) w d \mu_{N}, \forall v \in L^{r}(X)\right\} .
$$

If $\partial \Psi(u) \neq \emptyset$, then we say $\Psi$ is subdifferentiable at $u$.

Since $r<\infty$, it is well known that if $\Psi$ is norm continuous, then an application of the Hahn-Banach theorem implies $\partial \Psi(u) \neq \emptyset$. 


\section{The unconstrained minimization problem}

This section is devoted to the unconstrained problem (1.6). Let us fix some notation. Consider $f_{0} \in L^{p^{\prime}}(D)$, assumed to be a non-negative and non-trivial function. Here the set $D$ is assumed to be a smooth bounded domain in $\mathbb{R}^{2}$. We let $\mathcal{R}$ denote the rearrangement class generated by $f_{0}$. For $f \in L^{p^{\prime}}(D), u_{f} \in W_{0}^{1, p}(D)$, as before, denotes the unique positive solution of (1.4). Finally, we use $d x$ instead of $d \mu_{2}$, the two dimensional Lebesgue measure.

The first main result of this section is the following

Theorem 3.1. The unconstrained problem (1.6) has a unique solution $\hat{f} \in \mathcal{R}$. Moreover, there exists a decreasing function $\phi$ such that

$$
\hat{f}=\phi(\hat{u}), \quad \text { a.e. in } D,
$$

where $\hat{u}=u_{\hat{f}}$. The equation (3.1) is called the Euler-Lagrange equation for $\hat{f}$.

The second main result is

Theorem 3.2. Let $D$ be a disk centered at the origin with radius $a$. Then $\hat{f}$, the unique solution of (1.6), is radial, i.e. $\hat{f}$ is a function of $r=|x|$. Moreover, $\hat{f}$ is increasing in $r$.

To prove the above theorems we need the following basic result.

Lemma 3.3. The following statements are true.

(i) $\Phi_{p}$ is weakly sequentially continuous in $L^{p^{\prime}}(D)$.

(ii) $\Phi_{p}$ is strictly convex.

(iii) $\Phi_{p}$ is Gâteaux differentiable. Moreover, the Gâteaux derivative of $\Phi_{p}$ at $f$, denoted $\Phi_{p}^{\prime}(f)$, can be identified with $\frac{p}{p-1} u_{f}$.

Proof. (i) Let us consider $f_{n} \rightarrow f$ in $L^{p^{\prime}}(D)$. For simplicity, let us set $u_{n}=u_{f_{n}}$ and $u=u_{f}$. We claim

$$
(p-1) \Phi_{p}(f)+p \int_{D}\left(f_{n}-f\right) u d x \leq(p-1) \Phi_{p}\left(f_{n}\right) \leq(p-1) \Phi_{p}(f)+p \int_{D}\left(f_{n}-f\right) u_{n} d x .
$$

We only prove the first inequality in (3.2), since the second one can be proved similarly. To this end, we begin by observing that

$$
(p-1) \Phi_{p}(g)=\sup _{v \in W_{0}^{1, p}(D)}\left\{p \int_{D} g v d x-\int_{D}|\nabla v|^{p} d x\right\},
$$

for every $g \in L^{p^{\prime}}(D)$, and that,

$$
(p-1) \Phi_{p}(f)=p \int_{D} f u d x-\int_{D}|\nabla u|^{p} d x
$$


recalling that $u=u_{f}$. From (3.3), with $g=f_{n}$, we infer

$$
(p-1) \Phi_{p}\left(f_{n}\right) \geq p \int_{D} f_{n} u d x-\int_{D}|\nabla u|^{p} d x
$$

This last inequality lends itself to

$$
(p-1) \Phi_{p}\left(f_{n}\right) \geq p \int_{D}\left(f_{n}-f\right) u d x+p \int_{D} f u d x-\int_{D}|\nabla u|^{p} d x .
$$

Finally, (3.5) in conjunction with (3.4) yields the first inequality in (3.2).

From (3.2), it is clear that in order to complete the proof of part (i), it suffices to show

$$
\lim _{n \rightarrow \infty} \int_{D}\left(f_{n}-f\right) u d x=0 \quad \text { and } \quad \lim _{n \rightarrow \infty} \int_{D}\left(f_{n}-f\right) u_{n} d x=0
$$

The first limit in (3.6) follows from the weak convergence of $\left\{f_{n}\right\}$ in $L^{p^{\prime}}(D)$, since $u \in L^{p}(D)$. However, the verification of the second limit in (3.6) requires more work. To this end, let us recall

$$
\begin{cases}-\Delta_{p} u_{n}=f_{n} & \text { in } D \\ u_{n}=0 & \text { on } \partial D .\end{cases}
$$

Multiplying the differential equation in (3.7) by $u_{n}$, and integrating the result over $D$, yields

$$
\int_{D}\left|\nabla u_{n}\right|^{p} d x=\int_{D} f_{n} u_{n} d x
$$

An application of Hölder's inequality to the right hand side of (3.8), followed by the Poincaré inequality, leads to

$$
\int_{D}\left|\nabla u_{n}\right|^{p} d x \leq C\left\|f_{n}\right\|_{p^{\prime}}\left\|u_{n}\right\|_{W_{0}^{1, p}(D)},
$$

where $C$ is a universal positive constant. Whence, $\left\{u_{n}\right\}$ is a bounded sequence in $W_{0}^{1, p}(D)$. This, in turn, implies existence of a subsequence of $\left\{u_{n}\right\}$, still denoted $\left\{u_{n}\right\}$, and $w \in W_{0}^{1, p}(D)$, such that

$$
u_{n} \rightarrow w \quad \text { in } W_{0}^{1, p}(D) \quad \text { and } \quad u_{n} \rightarrow w \quad \text { in } L^{p}(D)
$$

Next we write

$$
\int_{D}\left(f_{n}-f\right) u_{n} d x=\int_{D}\left(f_{n}-f\right)\left(u_{n}-w\right) d x+\int_{D}\left(f_{n}-f\right) w d x
$$

The first term on the right hand side of (3.9) tends to zero because of Lemma 2.6. The second term in (3.9) also tends to zero because of weak convergence of $\left\{f_{n}\right\}$ in conjunction with the fact that $w$ belongs to $L^{p}(D)$. This completes the proof of part (i). Parts (ii) and (iii) have been proved in [14]. 
Proof of Theorem 3.1 We first relax the minimization problem (1.6), by extending the admissible set $\mathcal{R}$ to $\overline{\mathcal{R}}$, the weak closure of $\mathcal{R}$ in $L^{p^{\prime}}(D)$. Whence, we obtain

$$
\inf _{f \in \overline{\mathcal{R}}} \Phi_{p}(f) .
$$

Clearly, (3.10) is solvable, since $\Phi_{p}$ is weakly continuous, and $\overline{\mathcal{R}}$ is weakly compact. In addition, thanks to the strict convexity of $\Phi_{p}$, the solution to (3.10) is unique. Let us denote this unique solution by $\hat{f}$. We now proceed to prove that in fact $\hat{f} \in \mathcal{R}$. To this end, we recall the necessary condition satisfied by $\hat{f}$; namely,

$$
0 \in \partial \Phi_{p}(\hat{f})+\partial \xi_{\overline{\mathcal{R}}}(\hat{f})
$$

where $\xi_{\overline{\mathcal{R}}}$ stands for the indicator function supported on $\overline{\mathcal{R}}$; i.e.,

$$
\xi_{\overline{\mathcal{R}}}(g)= \begin{cases}0 & g \in \overline{\mathcal{R}} \\ \infty & g \notin \overline{\mathcal{R}}\end{cases}
$$

see [5] for details. Since $\Phi_{p}$ is differentiable, $\partial \Phi_{p}(\hat{f})=\left\{\Phi_{p}^{\prime}(\hat{f})\right\}$. On the other hand, from Definition 2.4, we infer

$$
\partial \xi_{\overline{\mathcal{R}}}(\hat{f})=\left\{w \in L^{p}(D): \xi_{\overline{\mathcal{R}}}(f) \geq \xi_{\overline{\mathcal{R}}}(\hat{f})+\int_{D}(f-\hat{f}) w d x, \forall f \in L^{p^{\prime}}(D)\right\} .
$$

Note that from (3.12), we infer that for $(w, f) \in \partial \xi_{\overline{\mathcal{R}}}(\hat{f}) \times \overline{\mathcal{R}}$ :

$$
\int_{D}(f-\hat{f}) w d x \leq 0 .
$$

Also, from (3.11) we deduce

$$
\Phi_{p}^{\prime}(\hat{f})+w=0,
$$

for some $w \in \partial \xi_{\overline{\mathcal{R}}}(\hat{f})$. The equation (3.14), in turn, implies

$$
\int_{D} \Phi_{p}^{\prime}(\hat{f})(f-\hat{f}) d x+\int_{D} w(f-\hat{f}) d x=0, \quad \forall f \in L^{p^{\prime}}(D) .
$$

Recalling $\Phi_{p}^{\prime}(\hat{f})=\frac{p}{p-1} \hat{u}$, in conjunction with (3.15) and (3.13), we obtain

$$
\int_{D}(f-\hat{f}) \hat{u} d x \geq 0, \quad \forall f \in \overline{\mathcal{R}}
$$

Whence, $\hat{f}$ minimizes the linear functional $L(h)=\int_{D} h \hat{u} d x$, relative to $h \in \overline{\mathcal{R}}$.

From the differential equation

$$
-\Delta_{p} \hat{u}=\hat{f}, \quad \text { in } D,
$$


coupled with Lemma 7.7 in [9], it follows that the graph of $\hat{u}_{S}$, the restriction of $\hat{u}$ to the set $S(\hat{f})=\{x \in D: \hat{f}(x)>0\}$, has no significant flat zones on $S(\hat{f})$. Therefore, if we denote by $\mathcal{R}_{S}$, the functions which are rearrangements of $f_{0}$ on $S(\hat{f})$, then by Lemma 2.2 we infer existence of a decreasing function $\phi_{S}$ such that $\phi_{S}\left(\hat{u}_{S}\right) \in \mathcal{R}_{S}$. We now proceed to extend $\phi_{S}$ to a decreasing function $\phi$ in such a way that $\phi(\hat{u}) \in \mathcal{R}$. Let us assume for the moment that this task has been accomplished. Then, from Lemma 2.3, it follows that $\phi(\hat{u})$ is the unique minimizer of the functional $L$, whence we must have $\hat{f}=\phi(\hat{u})$, which is the desired result.

We now come to the issue of extending $\phi_{S}$. This is done in two steps. The first step is to show that $\hat{u}$ achieves its smallest values on $S(\hat{f})$. To this end, it suffices to prove the following inequality

$$
\alpha \equiv e s s \sup _{S(\hat{f})} \hat{u} \leq e s s \inf _{S(\hat{f})^{c}} \hat{u} \equiv \beta,
$$

where $S(\hat{f})^{c}$ denotes the complement of $S(\hat{f})$. In order to prove (3.17), we assume it is false and will derive a contradiction. So let us suppose $\alpha>\beta$, for the moment. Whence, there exist constants $\gamma, \delta$, and sets $A \subseteq S(\hat{f}), B \subseteq S(\hat{f})^{c}$, both of positive measure, such that $\beta<\gamma<\delta<\alpha$, and

$$
\hat{u} \geq \delta \quad \text { on } A \quad \text { and } \quad \hat{u} \leq \gamma \quad \text { on } B .
$$

We may assume $|A|=|B|$, otherwise we consider subsets of $A$ or $B$, see [16]. Let $\eta: A \rightarrow B$ be a measure preserving bijection; such a map exists, see for example [16]. Next, we define a new function $\bar{f}$ as follows:

$$
\bar{f}(x)= \begin{cases}\hat{f}(x) & x \in(A \cup B)^{c} \\ \hat{f}(\eta(x)) & x \in A \\ \hat{f}\left(\eta^{-1}(x)\right) & x \in B\end{cases}
$$

Clearly $\bar{f}$ is a rearrangement of $\hat{f}$. Since $\hat{f} \in \overline{\mathcal{R}}$, it follows from Corollary 2.5 that $\bar{f} \in \overline{\mathcal{R}}$. Thus,

$$
\begin{aligned}
\int_{D} \bar{f} \hat{u} d x-\int_{D} \hat{f} \hat{u} d x & =\int_{A \cup B} \bar{f} \hat{u} d x-\int_{A \cup B} \hat{f} \hat{u} d x=\int_{B} \bar{f} \hat{u} d x-\int_{A} \hat{f} \hat{u} d x \\
& =\int_{B} \hat{f}\left(\eta^{-1}(x)\right) \hat{u} d x-\int_{A} \hat{f} \hat{u} d x=\int_{A} \hat{f}(x) \hat{u}(\eta(x)) d x-\int_{A} \hat{f} \hat{u} d x \\
& \leq(\gamma-\delta) \int_{A} \hat{f} d x<0,
\end{aligned}
$$

which contradicts the minimality of $\hat{f}$, relative to $\overline{\mathcal{R}}$.

In the second step, we give an explicit definition of the extended function. We denote the extended function by $\phi$, and define it as follows:

$$
\phi(t)= \begin{cases}\phi_{S}(t) & t<\alpha \\ 0 & t \geq \alpha\end{cases}
$$


where $\alpha$ is defined as in (3.17). Clearly, $\phi$ is decreasing, and $\phi(\hat{u}) \in \mathcal{R}$. Hence, the proof of the theorem is completed.

Remark 3.1. If $f_{0}=\chi_{D_{0}}$, the characteristic function of some measurable set $D_{0} \subseteq D$, then, from Theorem 3.1, we can deduce $\hat{f}=\chi_{\hat{D}}$, for some $\hat{D} \subseteq D$, satisfying $|\hat{D}|=\left|D_{0}\right|$. In addition, from (3.1), it follows that $\hat{D}=\{x \in D: \hat{u}(x)<\beta\}$, for some $\beta>0$. This, in turn, implies that $\hat{D}$ contains a layer around the boundary $\partial D$, since $\hat{u} \in C(\bar{D})$. If $D$ is simply connected, we can additionally show that $\hat{D}$ is connected. To see this, assume the contrary. So, we assume there is a component of $\hat{D}$, say $\mathcal{U}$, such that the intersection of $\partial \mathcal{U}$ and $\partial D$ is empty. Observe that, $\hat{u}=\beta$ on $\partial \mathcal{U}$. Thus $\hat{u}$ satisfies

$$
\begin{cases}-\Delta_{p} \hat{u}=\hat{f} & \text { in } \mathcal{U} \\ \hat{u}=\beta & \text { on } \partial \mathcal{U}\end{cases}
$$

Applying the strong maximum principle to (3.18), we find $\hat{u}>\beta$ in $\mathcal{U}$. This clearly contradicts the fact that $\hat{u} \leq \beta$ throughout $\hat{D}$, hence, $\hat{D}$ is connected. Since $\hat{D}=\{x \in D: \hat{u}(x)<\beta\}, \hat{u}$ satisfies

$$
\begin{cases}-\Delta_{p} \hat{u}=\chi_{\{\hat{u}<\beta\}} & \text { in } D \\ \hat{u}=0 & \text { on } \partial D .\end{cases}
$$

By setting $v=\beta-\hat{u}$, we derive

$$
\Delta_{p} v=\chi_{\{v>0\}}
$$

which is the one phase obstacle problem for the $p$-Laplacian operator. Through a private communication with $\mathrm{H}$. Shahgholian, we found that many questions related to the free boundary of (3.19) are yet to be settled, see [11] and [13]. However, when $p=2$, the free boundary of the problem (3.19) is extensively studied, see for example [15].

In order to prove Theorem 3.2, we need the following result.

Lemma 3.4. Let $f \in L^{p^{\prime}}(B)$, where $B$ is a ball centered at the origin. Let $R$ be a rotation map about the origin, i.e. $R(\theta)=\left(\begin{array}{cc}\sin \theta & -\cos \theta \\ \cos \theta & \sin \theta\end{array}\right)$, and let $f_{R}(x)=f(R x)$. Let $u \in W_{0}^{1, p}(B)$ and $v \in W_{0}^{1, p}(B)$ satisfy

$$
\begin{cases}-\Delta_{p} u=f & \text { in } B \\ u=0 & \text { on } \partial B\end{cases}
$$

and

$$
\begin{cases}-\Delta_{p} v=f_{R} & \text { in } B \\ v=0 & \text { on } \partial B\end{cases}
$$

respectively. Then $v(x)=u(R x)$, in $B$.

Proof. The proof is straightforward, hence omitted. 
Proof of Theorem 3.2 Let us first show that $\hat{f}$ is radial. To this end, we let $R$ be a rotation map about the origin. Also, we set $\hat{f}_{R}(x)=\hat{f}(R x)$. Let $\hat{u} \in W_{0}^{1, p}(D)$, and $v \in W_{0}^{1, p}(D)$, denote the solutions of (1.4), with $f=\hat{f}$ and $f=\hat{f}_{R}$, respectively. From Lemma 3.4, we infer $v(x)=\hat{u}(R x)$. Whence

$$
\Phi_{p}\left(\hat{f}_{R}\right)=\int_{D} \hat{f}_{R} v d x=\int_{D} \hat{f}(R x) \hat{u}(R x) d x=\int_{D} \hat{f} \hat{u} d x=\Phi_{p}(\hat{f}) .
$$

Thus, $\hat{f}_{R}$ is also a solution of (1.6). By uniqueness, we deduce $\hat{f}=\hat{f}_{R}$. Since $R$ is arbitrary, we infer $\hat{f}$ is radial.

To prove $\hat{f}$ is increasing, we first need to show that $\hat{u}$ is radial and decreasing. To this end, it suffices to show the boundary value problem

$$
\begin{cases}-\Delta_{p} u=\hat{f} & \text { in } D \\ u=0 & \text { on } \partial D\end{cases}
$$

has a radial solution. Thus, we need to prove the following initial value problem is solvable.

$$
-\frac{1}{r}\left(r\left|u^{\prime}\right|^{p-2} u^{\prime}\right)^{\prime}=\hat{f}(r), \quad u^{\prime}(0)=0, \quad u(a)=0 .
$$

By integrating the above ordinary differential equation from 0 to $r$, we derive

$$
r\left|u^{\prime}\right|^{p-2} u^{\prime}=-\int_{0}^{r} s \hat{f}(s) d s .
$$

Thus, $u^{\prime} \leq 0$, since $\hat{f} \geq 0$. Hence $u$ is decreasing, as expected. Now, integrating (3.23), from $r$ to $a$, yields

$$
u(r)=\int_{r}^{a}\left(\frac{1}{t} \int_{0}^{t} s \hat{f}(s) d s\right)^{\frac{1}{p-1}} d t .
$$

Therefore, the unique solution of (3.22) is radial.

Now we apply Theorem 3.1, which ensures $\hat{f}$ satisfies (3.1), for some decreasing function $\phi$. Therefore, $\hat{f}$ must be increasing. This completes the proof of the theorem.

\section{The constrained minimization problem}

In this section we prove the constrained problem (1.7) is solvable. But first we need some preliminaries. We fix a measurable set $K \subseteq D$. Let $f_{0} \in L^{p^{\prime}}(D)$ be a non-negative function and denote by $S\left(f_{0}\right)$, the support of $f_{0}$, i.e. $S\left(f_{0}\right)=\left\{x \in D: f_{0}(x)>0\right\}$. Note that the definition of support used here differs from the usual one. For simplicity we write $S$ instead of $S\left(f_{0}\right)$. We also assume the sets $S \cap K, S \backslash K, K \backslash S$ and $D \backslash(S \cup K)$ all have positive measures. 
Let us set $\epsilon=\int_{K} f_{0} d x$. The class of rearrangements of $f_{0}$ in $D$ is denoted $\mathcal{R}\left(f_{0}\right)$, which for simplicity we use $\mathcal{R}$ instead of $\mathcal{R}\left(f_{0}\right)$. Finally, we set

$$
\Lambda=\left\{f \in L^{p^{\prime}}(D): \int_{K} f d x=\epsilon\right\} .
$$

Observe that $\Lambda$ is an affine subspace of codimension one in $L^{p^{\prime}}(D)$.

We are now ready to state the main result of this section.

Theorem 4.1. Let $f_{0}, \mathcal{R}, D, K, S$ and $\Lambda$ be as described in the beginning of this section. Then, the constrained problem:

$$
\inf _{f \in \mathcal{R} \cap \Lambda} \Phi_{p}(f)
$$

has a unique solution $\tilde{f}$. Moreover, $\tilde{f}$ satisfies the following Euler-Lagrange equation:

$$
\tilde{f}=\phi\left(\tilde{u}+\lambda \chi_{K}\right), \text { a.e. in } D,
$$

for some $\lambda \in \mathbb{R}$, and a decreasing function $\phi$, unknown a priori. Here $\tilde{u}$ stands for the solution of (1.4), with $f=\tilde{f}$.

The following result will be used in the proof of Theorem 4.1.

Lemma 4.2. Let $\mathcal{R}$ and $\Lambda$ be as in Theorem 4.1. Then, $\overline{\mathcal{R} \cap \Lambda}=\overline{\mathcal{R}} \cap \Lambda$, where the bar indicates the weak closure in $L^{p^{\prime}}(D)$.

Proof. Since $\overline{\mathcal{R}} \cap \Lambda$ is weakly closed, it follows that $\overline{\mathcal{R} \cap \Lambda} \subseteq \overline{\mathcal{R}} \cap \Lambda$. To prove the reverse inclusion, we fix $g \in \overline{\mathcal{R}} \cap \Lambda$, and consider a weakly open subbasis $\mathcal{N}_{\varrho, l}(g)$, containing $g$. Whence,

$$
\mathcal{N}_{\varrho, l}(g)=\left\{f \in L^{p^{\prime}}(D):\left|\int_{D} l f d x-\int_{D} l g d x\right|<\varrho\right\},
$$

where $l \in L^{p}(D)$. We set $\mathcal{V}=\left\{f \in L^{p^{\prime}}(D): \int_{D} l f d x=\int_{D} l g d x\right\}$, which is an affine subspace of codimension one in $L^{p^{\prime}}(D)$, and observe that $\mathcal{V} \subseteq \mathcal{N}_{\varrho, l}(g)$. Let $\mathcal{K}=\mathcal{V} \cap \overline{\mathcal{R}} \cap \Lambda$, so $\mathcal{K}$ is convex, weakly compact and non-empty. Moreover, by the Krein-Milman theorem, see [6], we infer $\mathcal{K}=\overline{\operatorname{co}}(\operatorname{ext}(\mathcal{K}))$. Therefore, $\operatorname{ext}(\mathcal{K})$ is not empty. However, $\operatorname{ext}(\mathcal{K})=\mathcal{V} \cap \mathcal{R} \cap \Lambda$, by Lemma 2.1. Whence, $\mathcal{N}_{\varrho, l}(g) \cap \mathcal{R} \cap \Lambda$ is not empty, which implies $g$ must be a weak limit point of $\mathcal{R} \cap \Lambda$. Thus, $g \in \overline{\mathcal{R} \cap \Lambda}$, as desired.

Proof of Theorem 4.1 We begin by relaxing the problem (4.1). To this end, we extend $\mathcal{R} \cap \Lambda$ to $\overline{\mathcal{R} \cap \Lambda}$, and consider:

$$
\inf _{f \in \overline{\mathcal{R} \cap \Lambda}} \Phi_{p}(f) .
$$

Since $\overline{\mathcal{R} \cap \Lambda}$ is weakly compact, and $\Phi_{p}$ is weakly continuous, the minimization problem (4.1) is solvable. Moreover, $\overline{\mathcal{R} \cap \Lambda}=\overline{\mathcal{R}} \cap \Lambda$, by Lemma 4.2, hence $\overline{\mathcal{R} \cap \Lambda}$ is convex. This, along 
with the fact that $\Phi_{p}$ is strictly convex imply that (4.1) has a unique solution. Let us denote the solution by $\tilde{f}$. We claim that, in fact, $\tilde{f} \in \mathcal{R} \cap \Lambda$. To prove the claim, we first write the necessary condition satisfied by $\tilde{f}$ :

$$
0 \in \partial \Phi_{p}(\tilde{f})+\partial \xi_{\overline{\mathcal{R}} \cap \Lambda}(\tilde{f}),
$$

where $\xi_{\overline{\mathcal{R}} \cap \Lambda}$ denotes the indicator function supported on $\overline{\mathcal{R}} \cap \Lambda$. From (4.4), we infer existence of $g \in \partial \xi_{\overline{\mathcal{R}} \cap \Lambda}(\tilde{f})$ such that

$$
\frac{p}{p-1} \int_{D} \tilde{u}(f-\tilde{f}) d x+\int_{D} g(f-\tilde{f}) d x=0, \quad \forall f \in L^{p^{\prime}}(D),
$$

where we have used $\partial \Phi_{p}(\tilde{f})=\left\{\Phi_{p}^{\prime}(\tilde{f})\right\}$; here $\tilde{u}$ denotes the solution of (1.4), with $f=\tilde{f}$. From (4.5), we deduce

$$
\int_{D} \tilde{u}(f-\tilde{f}) d x \geq 0, \quad \forall f \in \overline{\mathcal{R}} \cap \Lambda .
$$

The inequality (4.6) implies that $\tilde{f}$ minimizes the linear functional $\tilde{L}(f)=\int_{D} \tilde{u} f d x$, relative to $f \in \overline{\mathcal{R}} \cap \Lambda$. At this stage we utilize Lemma 2.7. For this purpose, we set $l_{1}(f)=\int_{D} \chi_{K} f d x$, $l_{2}(f)=\tilde{L}(f), \mathcal{C}=\overline{\mathcal{R}}$ and $I=\epsilon$. In order to apply Lemma 2.7, we only need to verify existence of $f_{1}$ and $f_{2}$ in $\overline{\mathcal{R}}$ such that

$$
\int_{D} \chi_{K} f_{1} d x<\epsilon<\int_{D} \chi_{K} f_{2} d x
$$

We construct $f_{1}$ as follows. Let $A \subseteq S \cap K$ and $B \subseteq D \backslash(S \cup K)$ be measurable sets with $|A|=|B|$. Let $\eta_{1}: A \rightarrow B$ be a measure preserving bijection. Define

$$
f_{1}(x)= \begin{cases}f_{0}(x) & x \in(A \cup B)^{c} \\ f_{0}\left(\eta_{1}(x)\right) & x \in A \\ f_{0}\left(\eta_{1}^{-1}(x)\right) & x \in B\end{cases}
$$

Clearly, $f_{1} \in \mathcal{R}$, and $\int_{D} \chi_{K} f_{1} d x<\int_{D} \chi_{K} f_{0} d x=\epsilon$. Next, we construct $f_{2}$. Let $C \subseteq S \backslash K$ and $D \subseteq K \backslash S$ be measurable sets with $|D|=|C|$. Let $\eta_{2}: C \rightarrow D$ be a measure preserving bijection. Let

$$
f_{2}(x)= \begin{cases}f_{0}(x) & x \in(C \cup D)^{c} \\ f_{0}\left(\eta_{2}(x)\right) & x \in C \\ f_{0}\left(\eta_{2}^{-1}(x)\right) & x \in D .\end{cases}
$$

Then, $f_{2} \in \mathcal{R}$, and $\int_{D} \chi_{K} f_{2} d x>\int_{D} \chi_{K} f_{0} d x=\epsilon$. Now we can apply Lemma 2.7 to infer existence of $\lambda \in \mathbb{R}$ such that $\tilde{f}$ minimizes the linear functional $M(f)=\int_{D} f\left(\tilde{u}+\lambda \chi_{K}\right) d x$, relative to $\overline{\mathcal{R}}$. Observe that the restriction of $\tilde{u}$ to $S(\tilde{f})$ has no significant flat zones, hence the same holds for the restriction of $\tilde{u}+\lambda \chi_{K}$ to $S(\tilde{f})$. Similarly to the proof of Theorem 3.1 we can show that $\tilde{u}+\lambda \chi_{K}$ attains its smallest values on $S(\tilde{f})$ (see (3.17)), hence we can apply precisely 
the same argument as in Theorem 3.1 to deduce that there exists a decreasing function $\phi$ such that $\phi\left(\tilde{u}+\lambda \chi_{K}\right) \in \mathcal{R}$. Thus, by Lemma 2.3, we obtain

$$
\tilde{f}=\phi\left(\tilde{u}+\lambda \chi_{K}\right) \quad \text { a.e. in } D .
$$

This completes the proof of the theorem.

Remark 4.1. Interested readers are encouraged to use the ideas and tools presented in this paper to investigate the following maximization problem:

$$
\sup _{f \in \mathcal{R} \cap \Lambda} \mathcal{I}(f)
$$

where $\mathcal{I}(f)$ is defined as in (1.9). This problem will certainly be of interest to the authors of [8].

Acknowledgement. The authors wish to express their gratitude to the anonymous referee for his/her suggestions and comments which improved the presentation greatly.

\section{References}

[1] Burton, G. R., Rearrangements of functions, maximization of convex functionals and vortex rings. Math. Ann., 276 (2) (1987), 225-253.

[2] Burton, G. R., Variational problems on classes of rearrangements and multiple configurations for steady vortices. Ann. Inst. H. Poincaré Anal. Non Linéaire, 6 (4) (1989), 295-319.

[3] Burton, G. R.; McLeod, J. B., Maximisation and minimisation on classes of rearrangements. Proc. Roy. Soc. Edinburgh Sect. A, 119 (3-4) (1991), 287-300.

[4] Burton, G. R.; Ryan, E. P., On reachable sets and extremal rearrangements of control functions. SIAM J. Control Optim., 26 (6) (1988), 1481-1489.

[5] Clarke, F. H.; Ledyaev, Yu. S.; Stern, R. J.; Wolenski, P. R., Nonsmooth analysis and control theory. Graduate Texts in Mathematics, 178. Springer-Verlag, New York, 1998.

[6] Conway, J. B., A course in functional analysis. Second edition. Graduate Texts in Mathematics, 96. Springer-Verlag, New York, 1990.

[7] Cuccu, F.; Emamizadeh, B.; Porru, P., Nonlinear elastic membranes involving the $p$ Laplacian operator. Electron. J. Differential Equations, (49) (2006), 1-10. 
[8] Del Pezzo, L. M.; Fernández Bonder, J., Some optimization problems for $p$-Laplacian type equations. Appl. Math. Optim., 59 (3) (2009), 365-381.

[9] Gilbarg, D.; Trudinger, N. S., Elliptic partial differential equations of second order. Reprint of the 1998 edition. Classics in Mathematics. Springer-Verlag, Berlin, 2001.

[10] Henrot, A.; Maillot, H., Optimization of the shape and the location of the actuators in an internal control problem. Boll. Unione Mat. Ital. Sez. B Artic. Ric. Mat. (8), 4 (3) (2001), 737-757.

[11] Karp, L.; Kilpeläinen, T.; Petrosyan, A.; Shahgholian, H., On the porosity of free boundaries in degenerate variational inequalities. J. Differential Equations, 164 (1) (2000), 110-117.

[12] Kawohl, B., Rearrangements and convexity of level sets in PDE. Lecture Notes in Mathematics, 1150. Springer-Verlag, Berlin, 1985.

[13] Lee, K.; Shahgholian, H., Hausdorff measure and stability for the $p$-obstacle problem $(2<p<\infty)$. J. Differential Equations, 195 (1) (2003), 14-24.

[14] Marras, M., Optimization in problems involving the $p$-Laplacian. Electron. J. Differential Equations, (02) (2010), 1-10.

[15] Petrosyan, A.; Shahgholian, H.; Uraltseva, N., Regularity of free boundaries in obstacle-type problems. Graduate Studies in Mathematics, 136. American Mathematical Society, Providence, RI, 2012.

[16] Royden, H. L., Real analysis. Third edition. Macmillan Publishing Company, New York, 1988.

[17] Turkington, B.; Eydeland, A., An iterative method for computing steady vortex flow systems. Mathematical aspects of vortex dynamics (Leesburg, VA, 1988), 80-87, SIAM, Philadelphia, PA, 1989. 